\title{
5 Schlaf und Chronobiologie
}

\section{J. ZuLLEY}

\section{Die zirkadiane Periodik}

Unter natürlichen Bedingungen steht der Wechsel von Schlafen und Wachen im Einklang mit dem 24stündigen Wechsel von Tag und Nacht. Dies trifft auch für den tagesperiodischen Wechsel anderer physiologischer und psychologischer Variablen zu. Der Frage, inwieweit der 24-h-Tag bzw. die mit diesem Tag variierenden Umweltfaktoren diese Rhythmen erzeugen, wurde durch Experimente nachgegangen, die diese Einflußgrößen ausschlossen. In Humanexperimenten erreicht man dies durch einen längeren Aufenthalt (ca. 4 Wochen) freiwilliger Versuchspersonen in einem von der Umwelt isolierten unterirdischen Versuchsraum [9]. Die Versuchspersonen leben hier ohne sozialen Kontakt (außer schriftlichem) und können somit ihr Schlaf-Wach-Verhalten vollständig den eigenen Bedürfnissen anpassen. Der Schlaf-Wach-Wechsel wie auch andere psychologische und physiologische Funktionen verliefen in diesen Experimenten weiterhin regelmäßig und ungedämpft weiter (Abb. 1). Diese sog. freilaufenden oder auch autonomen zirkadianen (circa - dies; etwa 24 h) Rhythmen zeigten in der überwiegenden Mehrzahl eine mittlere Periode von mehr als $24 \mathrm{~h}$ (im Mittel 25,0 h). Da diese Periodenlänge in der natürlichen Umwelt nicht vorkommt, wurde vor allem hieraus geschlossen, daß ein endogenes System für die zirkadianen Schwankungen der Variablen verantwortlich ist. Als eine wichtige Hirnstruktur, die für die zirkadiane Rhythmizität mitverantwortlich ist, wurde in Tierversuchen der Nucleus suprachiasmaticus ( $\mathrm{SCN}$ ) im ventralen Hypothalamus lokalisiert [2].

Als Modell wird angenommen, daß ein System gekoppelter Oszillatoren den zeitlichen Ablauf der verschiedenen Variablen steuert und sie im Hinblick auf eine optimale Funktionalität koordiniert.

Verschiedene Modelle werden diskutiert, die sich vor allem durch die Anzahl der postulierten inneren „Uhren“ unterscheiden, wobei das Spektrum vom „EinOszillator-Modell“ bis zum „Multioszillatorenmodell“ [9] reicht. In letzter Zeit wird vor allem das „Zwei-Prozeß-Modell“" von Borbély [3, 4] diskutiert, ein EinOszillator-Modell, welches neben einem zirkadianen Faktor (Faktor C) auch den Einfluß eines homöostatischen Faktors (S) im Sinne einer Schlafsubstanz annimmt.

Die zirkadiane Periodik wird durch innere Uhren erzeugt, sie kann jedoch durch entsprechend periodisch auftretende Außenreize (Zeitgeber) auch auf bestimmte andere Periodenwerte (Mitnahmebereich) synchronisiert werden. 


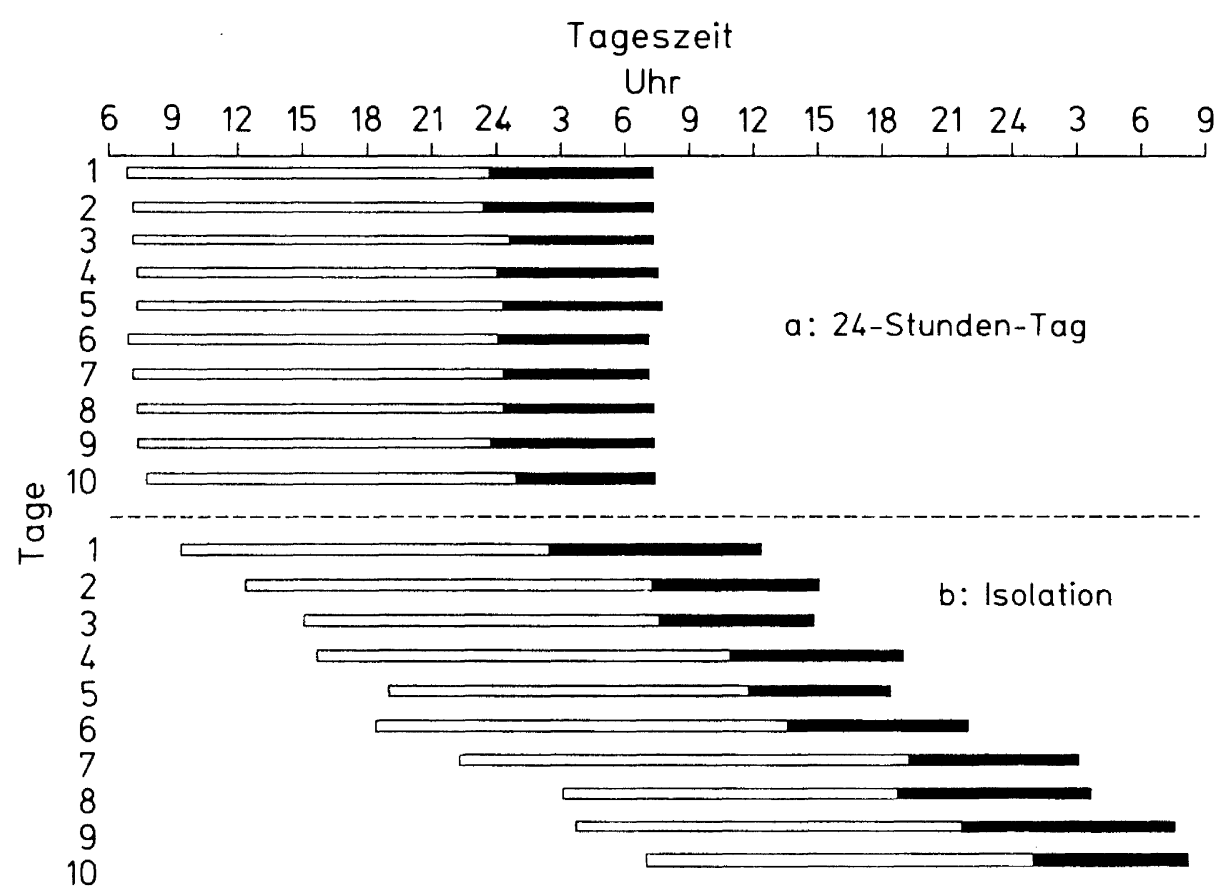

\begin{abstract}
Abb. 1 a, b. Aufeinanderfolgende Tage (untereinandergezeichnet) einer Versuchsperson. a Im normalen 24-h-Tag und $\mathbf{b}$ unter Isolationsbedingungen mit einer freilaufenden zirkadianen Periodik (offene Balken Wachzeit, schwarze Balken Schlafzeit). Die Verlängerung des Schlaf-WachRhythmus zeigt sich in der Verschiebung der Schlaf-Wach-Balken. (Daten aus [11])
\end{abstract}

Unter natürlichen Lebensbedingungen stammen diese Zeitgeber aus der Umwelt (z.B. die mit dem Tag-Nacht-Wechsel verbundenen Licht- und Temperaturveränderungen) und synchronisieren die zirkadianen Rhythmen auf $24 \mathrm{~h}$. Beim Menschen scheint jedoch die ,soziale Relevanz“ solcher Zeitgeberreize ausschlaggebend für deren synchronisierende Wirkung zu sein.

In Experimenten wurden durch künstliche Zeitgeber (z. B. vorgegebene HellDunkel-Wechsel) die Grenzen der Synchronisierbarkeit (Mitnahmebereich) für die verschiedenen Variablen festgestellt. Auf diese Weise konnte die unterschiedliche Stärke verschiedener Zeitgeber untersucht werden.

Beim Menschen erwies sich ein reiner Hell-Dunkel-Wechsel mit normaler Raumbeleuchtung (etwa 400 Lux) als schwacher Zeitgeber. Erst wenn zusätzliche Informationen (regelmäßige Gongsignale am Tage) gegeben wurden, konnten die physiologischen Variablen auf Werte zwischen 23 und 27 h synchronisiert werden. Weit größer wird der Mitnahmebereich, wenn die Beleuchtungsstärke die Intensität von hellem Tageslicht ( $>2000$ Lux) hat [10].

Die Körpertemperatur als Repräsentant der physiologischen Funktionen und der Schlaf-Wach-Wechsel als Verhaltensparameter stellen die bedeutendsten Meßgrößen in der Human-Chronobiologie dar. Im Freilauf (s. oben) verlaufen sie in der Mehrzahl der Fälle synchron (interne Synchronisation), in etwa $30 \%$ der Fälle dagegen kommt es zur internen Desynchronisation.

- Bei interner Synchronisation haben alle zirkadianen Rhythmen die gleiche Periodenlänge bei fester Phasenbeziehung. Dies zeigt sich im normalen Alltag 
durch eine feste Lage des Minimums der Körpertemperatur in der zweiten Hälfte der Schlafzeit. Entsprechendes gilt für die anderen zirkadianen Rhythmen.

- Ein Übergangsstadium zwischen interner Synchronisation und Desynchronisation stellt die interne Dissoziation dar. Hierbei kommt es vorübergehend zu einer Phasenverschiebung mit anschließender Wiederherstellung einer festen Phasenbeziehung (z. B. Jet-lag).

- Interne Desynchronisation ist ein Zustand, in dem verschiedene interne Rhythmen ihre Phasenbeziehungen systematisch ändern (bestimmte Zustände der verschiedenen Schwingungen kommen nicht gleichzeitig vor); sie treten hierbei mit unterschiedlicher Periodenlänge auf. Der periodische Verlauf der Körpertemperatur bleibt in diesem Fall im zirkadianen Bereich, während der SchlafWach-Rhythmus erheblich abweichen kann. Zu unterscheiden ist zwischen einer Verlängerungsdesynchronisation (Schlaf-Wach-Periode nimmt Werte bis zu $50 \mathrm{~h}$ an) und einer Verkürzungsdesynchronisation (Schlaf-Wach-Periode nimmt Werte bis zu $12 \mathrm{~h}$ an). Bisher wurde dieses Phänomen nur beim Menschen gefunden. Möglicherweise handelt es sich hierbei um ein Abweichen des „subjektiven Tages" und nicht einer zugrundeliegenden physiologischen SchlafWach-Rhythmik von der zirkadianen Periodik [13].

Diese Befunde stützen die Annahme, daß dem Schlaf-Wach-Wechsel innerhalb des zirkadianen Systems eine eher untergeordnete Rolle zukommt. Beim Menschen besteht die Möglichkeit, sowohl experimentell wie auch willkürlich den Schlaf-Wach-Rhythmus von der inneren Uhr abzukoppeln. Der Wechsel von Schlafen und Wachen ist dann nicht mehr Ausdruck des zugrundeliegenden zirkadianen Systems. Die Anbindung des Schlaf-Wach-Wechsels an den zirkadianen Oszillator zeigt sich dann aber weiterhin in der Bevorzugung bestimmter Einschlafzeitpunkte innerhalb der zirkadianen Periodik und in der Abhängigkeit der Schlafdauer von diesem Einschlafzeitpunkt [12]. Eine weitere Abhängigkeit des Schlafes vom zirkadianen System spiegelt sich auch in der Schlafstadienstruktur wider: das Auftreten von REM-Schlaf unterliegt einer zirkadianen Schwankung [11].

\section{Ultradiane Periodik}

Sowohl vom experimentellen Ansatz wie auch von der Datenauswertung her lag das Schwergewicht in der bisherigen Forschung auf Periodenlängen um $24 \mathrm{~h}$ (zirkadiane Periodik). Dabei wurden kürzere (ultradiane) Periodenwerte zwar bemerkt, jedoch in der weiteren Bearbeitung kaum berücksichtigt. Dies trifft für physiologische Variablen wie Körpertemperatur und Kortisol und für psychologische Meßgrößen wie subjektive Wachheit und Leistungsfähigkeit ebenso zu wie für das Schlaf-Wach-Verhalten. Der Zustand des menschlichen Organismus zeigt somit am Tage mehrere deutliche Schwankungen, die sich auch in veränderter Leistungsfähigkeit und Müdigkeit ausdrücken. Als Beispiel sei hier die orthosta- 
tische Kreislaufreaktion genannt. Die Untersuchungen der orthostatischen Labilität ergaben zwei Maxima innerhalb eines 24-h-Tages. Neben einem deutlich ausgeprägten Maximalwert gegen 3 Uhr morgens zeigte sich auch ein Maximum gegen Mittag [1].

Im Unterschied zur Untersuchung zirkadianer Aspekte ist es bei der Analyse ultradianer Verläufe erforderlich, die Daten in einem schmaleren Zeitraster zu erheben, das Spontanverhalten der Versuchspersonen mit möglichen kurzfristigen Änderungen ausdrücklich zuzulassen und bei der Datenverarbeitung die entsprechend kurzen Periodenwerte zu analysieren.

In den folgenden Experimenten, in welchen das spontane Schlaf-Wach-Verhalten untersucht wurde, lebten die Versuchspersonen isoliert von der Umwelt, wie in den Standardexperimenten der Chronobiologie. Zusätzlich hatten die Versuchspersonen keinerlei Beschäftigungsmaterial, ihnen wurden keine Anweisungen über ihr Schlafverhalten gegeben und sie sollten ihren Tagesablauf nicht strukturieren. Außerdem konnten sie im Gegensatz zu den bisherigen Untersuchungen keine koffeinhaltigen oder alkoholische Getränke zu sich nehmen. Die Ergebnisse zeigten, daß sich unter diesen monotonen Bedingungen das unimodale Schlaf-Wach-Muster in eine bimodale Verteilung der Häufigkeit der Schlafepisoden über den ,zirkadianen“ Tag verändert [5]. Dies bedeutet, daß neben der Hauptschlafphase während der Nachtzeit sich unter diesen Bedingungen auch ein zweiter bevorzugter Zeitpunkt für Schlaf zeigt. Er ist gegenüber der Hauptschlafphase um ca. 12 Stunden verschoben (Abb. 2a). Als Modellannahme läßt sich eine „Schlafschwelle“ beschreiben. Wird diese „Schlafschwelle“ gesenkt, d. h. die Wahrscheinlichkeit für Schlaf erhöht, so drückt sich dies im Auftreten einer zweiten Schlafepisode mit einer bestimmten bevorzugten Phasenlage aus, wobei sich dann für das gesamte Schlaf-Wach-Muster eine ca. 12stündige Schlaf-WachPeriodik zeigt [6].

Ein zirkadianer Einfluß ist weiterhin in der Schlafdauer zu sehen, der Nachtschlaf ist deutlich länger als der Tagschlaf, der sich in seiner Struktur ansonsten nicht vom Nachtschlaf unterscheidet. Dies entspricht den bisherigen Befunden über den Zusammenhang zwischen Schlafdauer und Zeitpunkt des Schlafbeginns [12].

Das Auftreten einer zweiten Schlafphase entspricht im Alltag dem Mittagsschlaf. Dessen Auftreten scheint davon abzuhängen, ob die Umweltbedingungen eine solche Ruhepause zulassen. Kinder, Studenten und ältere Menschen zeigen in der Mehrzahl einen Mittagsschlaf. Ebenso gehört ein Mittagsschlaf in vielen nichtindustrialisierten Kulturen zum Alltag. In Isolationsuntersuchungen zeigte sich ein deutlicher Anstieg der Tagschlafhäufigkeit, wenn es den Probanden völlig freigestellt war, wann sie schliefen. Diese Befunde weisen darauf hin, daß diese zweite bevorzugte Schlafphase zwar biologisch verankert ist, beim erwachsenen Menschen aber durch Umwelteinflüsse oder willkürliche Verhaltenskontrolle übersprungen werden kann.

Ein weiterer Beleg für eine vorgegebene zweite Ruhephase ist der Befund, daß viele psychologischen und physiologischen Variablen zu diesem Zeitpunkt auch ohne Mittagsschlaf einen ähnlichen Verlauf zeigen wie während des zweiten Teils der Nachtzeit. Eine Verringerung der Leistungsfähigkeit, größere Fehlerra- 
ten, verstärkte Müdigkeit sowie eine Absenkung der Körpertemperatur unabhängig von einer Mahlzeiteinnahme zeigen an, daß der Organismus einen ähnlichen Umstellungsprozeß wie in der zweiten Nachthälfte erfährt. Der Gesamtorganismus ist demnach von seiner Kapazität her grundsätzlich nicht auf eine lange Aktivitätsdauer und eine einzige Ruhephase innerhalb eines zirkadianen Zyklus eingestellt, sondern zeigt innerhalb der Aktivitätsphase zumindest einen Wechsel in die trophotrope Phasenlage. Dieser Zeitpunkt, der beim Erwachsenen normalerweise problemlos ohne Schlaf überwunden werden kann, wird z. B. dort relevant, wo ein größeres Schlafbedürfnis vorhanden ist (Schichtarbeit, Zeitzonenflüge, Belastungsbedingungen). Es stellt sich jedoch die Frage, ob diese bimodale Schlafverteilung das eigentlich grundlegende Schlaf-Wach-Muster ist und wie sich dieses Muster bei experimenteller stufenweiser Veränderung der Schlafmöglichkeit und des Schlafbedürfnisses ändert.

In einer weiteren Versuchsserie [14] wurde die „Schlafschwelle“ gegenüber dem oben geschilderten Experiment noch weiter gesenkt. Die Versuchsperson befand sich allein in einem von der Umwelt abgeschirmten Raum, ohne die Möglichkeit einer Beschäftigung nachgehen zu können. Zusätzlich hatte sie während des gesamten Zeitraumes von 32 h kontinuierlich im Bett zu liegen.

Die Ergebnisse zeigten, daß neben den Hauptschlafphasen während der Nächte auch tagsüber vermehrt geschlafen wurde. Der Tagschlaf wies hierbei einen Tagesgang auf, der zusätzlich eine ultradiane Modulation zeigte. Der zirkadiane Anteil drückt sich in einem Abfall der Schlafhäufigkeit über den Tag hinweg, mit einer Phase deutlich verminderter Schlafbereitschaft um 19.00 Uhr, aus. Dieses Ergebnis entspricht den Befunden aus der Literatur, in denen für

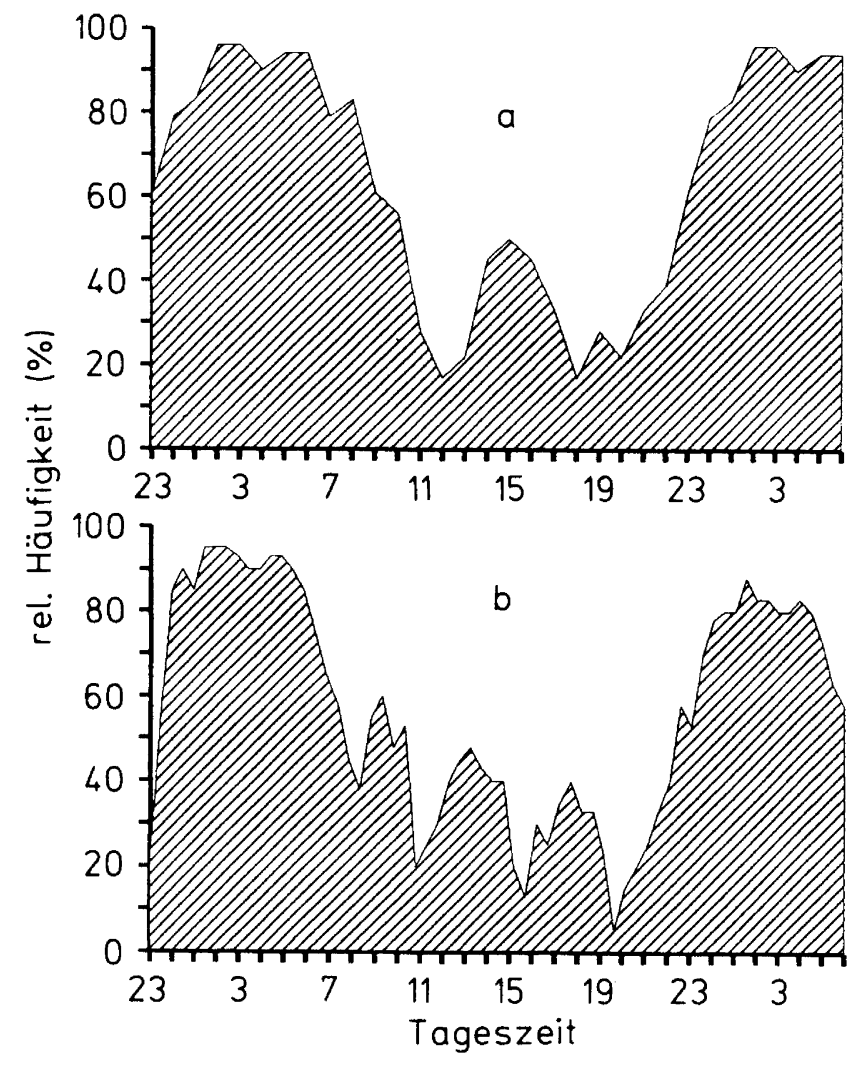

Abb. 2 a, b. Verteilung der Schlafhäufigkeit über den Versuchszeitraum von $32 \mathrm{~h}$ (Anteil schlafender Versuchspersonen an der Gesamtstichprobe; Stundenmittelwert). a 10 Versuchspersonen unter „Disentrainment"-Bedingungen (erzwungene Untätigkeit) (nach [5]). b 40 Versuchspersonen unter „Bettruhe“Bedingungen (erzwungene Untätigkeit bei konstanter Bettruhe) (siehe [14]) 
diesen Zeitraum eine „forbidden zone of sleep“ angegeben wird [8]. Dieser Abfall zeigt auch, daß die zirkadiane Verteilung von Schlafen und Wachen sich nicht nur auf die monophasische Plazierung des Nachtschlafes beschränkt, sondern sich auch in der Veränderung der Schlafbereitschaft während des Tages ausdrückt. Das ultradiane Muster zeigt eine Bevorzugung bestimmter Tageszeiten für das Auftreten von Schlaf (9.00,13.00 und 17.00 Uhr) (Abb. 2b). Der zeitliche Abstand dieser Schlafgipfel deutet eine 4-h-Periodik von Schlafen und Wachen an.

Interpretieren lassen sich die experimentellen Ergebnisse unter der Annahme einer weiteren Absenkung der „Schlafschwelle“. Das Ergebnis dieser zusätzlichen Absenkung ist das Auftreten zweier dem „Mittagsschlaf“ benachbarter Phasen bevorzugten Einschlafens im Abstand von jeweils $4 \mathrm{~h}$. Eine durch die Lebensbedingungen erfolgte Absenkung der „Schlafschwelle“ findet sich bei Kleinkindern und auch bei pathologischen Zuständen im hohen Alter. Bei diesen Personenkreisen wurde ebenfalls von einer 4-h-Periodik berichtet [7].

Ein weiterer Hinweis auf das Vorhandensein einer 4-h-Periodik ist der Befund, daß die Schlafdauer in diesen Untersuchungen eine zweigipflige Verteilung zeigt. Kurze Schlafepisoden haben eine mittlere Dauer von 1,5 h und lange Schlafepisoden von 7,5 h. Schlafepisoden mit einer Dauer zwischen 4-5 h scheinen eine seltene Ausnahme zu sein. Dieses Ergebnis kann dahingehend interpretiert werden, daß während des Schlafes eine Bereitschaft zu erwachen besteht, die nach $4 \mathrm{~h}$ ein Maximum erreicht und somit als Bestandteil der 4stündigen Schlaf-WachRhythmik anzusehen ist.

Insgesamt zeigen die Untersuchungen, daß der Zustand des menschlichen Organismus bei erhöhter Müdigkeit einer 12stündigen und bei weiter zunehmendem Schlafbedarf einer 4stündigen Periodik folgt. Diese ultradiane Periodik scheint die zeitliche Grundstruktur der Schlafbereitschaft des Organismus zu sein.

\section{Literatur}

1. Aschoff J, Aschoff J (1969) Tagesperiodik der orthostatischen Kreislaufreaktion. Pflügers Arch 306: 146-152

2. Aschoff J, Daan S, Groos GA (eds) (1982) Vertebrate circadian systems. Springer, Berlin Heidelberg New York

3. Borbély A (1982) A two process model of sleep regulation. Hum Neurobiol 1: 195-204

4. Borbély A (1987) Das Geheimnis des Schlafs. dtv, München

5. Campbell S, Zulley J (1985) Ultradian components of human sleep/wake patterns during disentrainment. In: Experimental brain research, Suppl 12. Springer, Berlin Heidelberg New York Tokyo, pp 234-254

6. Campbell S, Zulley J (1989) Napping in time-free environments. In: Dinges DF, Broughton RJ (eds) Sleep and alertness. Raven Press, New York, pp 121-138

7. Dinges DF, Broughton RJ (eds) (1989) Sleep and alertness. Raven Press, New York

8. Lavie P, Scherson A (1981) Ultrashort sleep-waking schedule. I. Evidence of ultradian rhythmicity in »sleepability«. EEG Clin Neurophysiol 52: 163-174

9. Wever R (1979) The circadian system of man. Springer, New York

10. Wever R (1989) Light effects on human circadian rhythms: A review of recent Andechs experiments. J Biol Rhythms 4: 161-185 
11. Zulley J (1979) Der Einfluß von Zeitgebern auf den Schlaf des Menschen. Rita G. Fischer, Frankfurt

12. Zulley J, Wever R. Aschoff J (1981) The dependence of onset and duration of sleep on the circadian rhythm of rectal temperature. Pflügers Arch 391: 314-318

13. Zulley J, Campbell S (1985) Napping behavior during ..spontaneous internal desynchronisation": Sleep remains in synchrony with body temperature. Hum Neurobiol 4: 123-126

14. Zulley J, Bailer J (1988) Polyphasic sleep/wake pattern and their significance to vigilance. In: Leonhard JP (ed)Vigilance: Methods, models and regulation. Lang, Frankfurt, pp 167180 